\title{
The Effectiveness of Autograft Used in Anterior Cruciate Ligament Reconstruction of the Knee: Surgical Records for the New Generations of Orthopedic Surgeons and Synthetic Graft Revisit
}

This article was published in the following Dove Press journal:

Orthopedic Research and Reviews

\author{
Khaldoon M Bashaireh (D) \\ Ziad Audat (D) \\ Ahmad M Radaideh' \\ Abdelwahab J Aleshawi ${ }^{2}$ \\ 'Department of Special Surgery, Division \\ of Orthopedics, Faculty of Medicine, \\ Jordan University of Science and \\ Technology, Irbid 22II0, Jordan; ${ }^{2}$ Faculty \\ of Medicine, Jordan University of Science \\ and Technology, Irbid 221 I0, Jordan
}

\begin{abstract}
Objective: In this article, we aim to revisit the synthetic graft and review the advantages and disadvantages between different types of grafts for patients who underwent anterior cruciate ligament $(\mathrm{ACL})$ reconstruction in a tertiary medical institute for the new generations of surgeons.

Patients and Methods: Retrospectively, we identified 115 patients who underwent arthroscopic ACL reconstruction between 2006 and 2009. We were able to retrieve 74 patients from them. The 74 patients were divided into 32 patients who underwent primary arthroscopic ACL reconstruction with hamstring and patellar tendon autograft and 42 cases with an active biosynthetic composite $(\mathrm{ABC})$ ligament. The mean the follow-up period for both groups was 7 years. The following information was obtained: standard demographic information (age, sex), clinical presentation, presence of trauma, associated injuries, types of grafts (autograft versus synthetic graft) and postoperative complications. Moreover, functional and clinical outcomes in addition to the satisfaction of patients using the international knee documentation committee (IKDC) score and knee injury and osteoarthritis outcome score (KOOS) were measured.
\end{abstract}

Results: We found that the natural (autograft) was better in terms of clinical and functional outcome than the synthetic one (the scores of KOOS and IKDC were better in natural grafts). Furthermore, the immediate postoperative results for the pivot and Lachman tests were better in natural grafts. On the other hand, the rate of re-rupture was similar for both groups. However, the long-term inflammatory changes and stiffness that is attributed to the immunological reactions were more in the synthetic grafts.

Conclusion: This study revisited the synthetic graft and provided evidence that the natural grafts are more beneficial with less complications, as they had better immediate and longterm postoperative clinical and functional outcomes. We recommend the utilization of autograft as first choice and the synthetic not to be used given the current criteria.

Keywords: anterior cruciate ligament (ACL), arthroscopy, autologous, knee

\section{Introduction}

The anterior cruciate ligament (ACL) is important to maintaining the stability of the knee joint. ${ }^{1,2}$ It is crucial to identify and treat ACL tears, especially in the young, active population in whom surgical reconstruction is the best management option available. Controversy continues about which type of graft should be used during
Correspondence: Khaldoon M Bashaireh of Orthopedics, Faculty of Medicine, Jordan University of Science and

Technology, P. O. Box 3030, Irbid 22110, Jordan

Tel +962799051087

Fax +96227201064

Email bashaireh@just.edu.jo 
a reconstruction. $^{3-10}$ The ideal graft for ACL reconstruction would consist of the following: reproduction of the histological and biomechanical characteristics of the native ligament; fully and quickly incorporated within bone tunnels; no risk of rejection or disease transmission; minimal donor-site morbidity; be of sufficient length and diameter; and be cost-effective as well as readily available. ${ }^{11}$ Currently there are three types of grafts: autogenous, allograft and synthetic. ${ }^{11}$

There is general agreement on the use of the autogenous graft as first line option during the ACL reconstruction surgeries as this type of graft is the closest to the aforementioned ideal graft. ${ }^{12-14}$ There are several options for autogenous graft including hamstring tendons, bone-patellar tendonbone (BTB), and iliotibial tract. ${ }^{12}$ However, there are some disadvantages concerning their use, especially the effects on the donor site such as pain, crepitus and weakness of the extensor mechanism and some specific complications for BTB, such as patella fracture. ${ }^{13}$ Not forgetting the need for a well-trained, experienced surgeon, as well as the need for an extra step during surgery, thus requiring longer anesthetic and tourniquet times. ${ }^{14}$

Regarding the allograft, it is technically easier and does not require the extra step or the expert surgeon. However, it has many adverse effects. Firstly, it carries a higher risk of infection transmission (Hepatitis $\mathrm{C}$ and Band human immunodeficiency viruses) so special sterilization techniques with radiation or freezing are needed and this may affect the strength of the graft and add to their already higher cost. In addition, there are many studies that reported higher risk of re-rupture in young athletes. ${ }^{9,11,15}$

Due to the above-mentioned drawbacks; synthetic ligaments for ACL reconstruction like active biosynthetic composite (ABC; Surgicraft Ltd, Redditch, UK), which is composed of polyester and carbon fibers combined in a partial braid, were widely used in the late 1980s and early 1990s. The initial positives about the introduction of synthetic graft materials is regarding the lack of donor site morbidity, their abundant supply and their significant strength. $^{4-7}$ In a long-term follow-up, new issues were appearing; infections from allogenic material, an immunological response against the artificial ACL, tunnel osteolysis, femoral and tibial fractures near the tunnels, foreign-body synovitis and knee osteoarthritis were reported. ${ }^{8-10,16}$ Therefore, their use has decreased and almost disappeared during recent years. Concerns regarding the potential return to popularity of synthetic grafts for ACL reconstruction may arise. So, it is important to look back retrospectively and try to find out and review our past experience with different types of grafts especially the synthetic ones. In this article, we aim to present the advantages and disadvantages between different types of grafts for patients who underwent ACL reconstruction in a tertiary medical institute.

\section{Patients and Methods}

This study was conducted at a tertiary medical institute. After obtaining the Institutional Review Board (IRB) approval, we retrospectively identified 115 Patients who underwent ACL reconstruction between 2006 and 2009. Of these, only 74 patients could be recalled for retrospective review. The following information was obtained: standard demographic information (age, sex), clinical presentation, presence of trauma, associated injuries, types of grafts and postoperative complications. Moreover, functional and clinical outcomes in addition to the satisfaction of the patients using the IKDCand KOOS scores were measured.

The inclusion criteria for this study consisted of traumatic ACL ruptures, with or without meniscal lesions or with posterior cruciate ligament (PCL) injury. Exclusion criteria included patients with other ligament injuries, for example, associated fractures of the knee or post-knee dislocation, and with comorbidities such as cardiovascular diseases that could interfere with post-surgical rehabilitation.

\section{Setting}

All the ACL reconstruction operations were performed by the same expert orthopedic surgery team who follow the same surgical approach. All biological grafts were autologous. One source was dealt with for the synthetic grafts which is the ABC purely polyester ligament. An arthroscopic surgical procedure was performed for the reconstruction of the ACL in all patients under general anesthetic and using a tourniquet. For the quadruple hamstring autografts, a $2-3 \mathrm{~cm}$ incision is placed along the pes anserinus midway between the tibial tubercle and the posteromedial border of the tibia. A midline incision from the inferior pole of the patella reaching the tibial tuberosity for bone-patellar tendon-bone graft is made. The femoral tunnel was created using an anteromedial portal technique. A guide pin was drilled from the isometric point across the femur and out the lateral thigh, and the tunnel was over-drilled to the appropriate size and length. The tibial tunnel guide pin was placed at the center of the ACL footprint using an ACL guide set at approximately $45^{\circ}$ to the tibial plateau in the sagittal plane. The guide pin was over-drilled to the appropriate size. In the 
$\mathrm{ABC}$ graft, a second incision was made over the lateral aspect of the distal femur, anterior to the intermuscular septum, then a 2- to 3-mm groove was created in the overthe-top position with a rasp, the graft was introduced through the tibial tunnel and from the over-the-top femur position in the groove, and was fixed at both ends with a bollard. Fixation was done using absorbable screws for natural grafts. The knee was assessed immediately postreconstruction by examining it. The Lachman test and pivot shift test gave an idea about the stability of the knee both pre- and postoperatively. Reconstruction of the ligament was followed by a vigorous rehabilitation program with both active and passive knee motion. On average the patientremained in hospital for 4 days after the operation for supervised physiotherapy. Later, with a mean follow up of 7 years post-reconstruction, we assessed the functional and clinical outcomes in addition to satisfaction of the patients, using the IKDC and KOOS scores.

During the period 2006-2009, 3 types of graft were used during ACL reconstructions. Two biological grafts and one synthetic. The first is the quadruple hamstring tendon graft (Gracillis and Semtendinosus muscle tendons). The second is the BTB graft. The third is the synthetic artificial ligament which is constructed from a partial polyester braid over a core of carbon polyester. It has a tensile strength of $3.13 \mathrm{KN}$, which is greater than the strength of the natural ACL $(2.50 \mathrm{KN})$. The implant had radial over braiding at both ends, with integral bollard fixation, and had been designed to act as a scaffold, stimulating tissue cover and ingrowth into the implant.

\section{Statistical Analysis}

Data were entered into a spreadsheet. Statistical analyses were performed using IBM SPSS Statistics Software (v.21), 2012. Data were presented as frequency distributions for categorical variables and mean \pm standard error of the mean (SE) for continuous variables. Data was tested at a significance level of $0.05 \%$. The Pearson $\chi 2$ test was used to investigate the significance of association between categorical variables, while Student's $t$-test was applied to examine the significance level for continuous normally distributed variables. The $P<0.05$ is the significant relationship between variables.

\section{Results}

The study included 74 individuals (68 males and 6 females). Most of these individuals were not athletes. The mean age of the individuals was 30.4 years. The youngest was 17-years- old and the oldest was 51-years-old. The male predominance reflects the social status which demonstrates that males carry out the heavier occupations (see Table 1).

Trauma is the leading cause for ACL injury in our sample. Only 5 individuals were not traumatic and two of them were epilepsy patients. The most common clinical presentation was giving way and pain. Table 1.

An arthroscopy of the injured knee was done prior to reconstruction to confirm the ACL rupture and to look for associated symptoms. We noticed that 43 patients had associated injuries. The most frequent associated injury that was detected was a medial meniscus tear as it occurred in 28 individuals. All meniscal tears were treated simultaneously by partial meniscectomy. Only one patient had undergone both ACL and PCL reconstruction at two-staged operations. All reconstructions were done arthroscopically.

Time from trauma to surgery was variable, but most surgeries were performed before 24 months had passed. The mean time was 40 months and the median was 18 months. The shortest period was 2 month and longest was 24 years Table 1.

Table I Patients Characteristics and General Frequencies

\begin{tabular}{|c|c|c|}
\hline General Frequencies & & \\
\hline Sex & Number & Percent \\
\hline Male & 68 & 91.9 \\
\hline \multirow[t]{2}{*}{ Female } & 6 & 8.1 \\
\hline & Mean & SE \\
\hline Age & 30.4 & 1.0 \\
\hline \multirow[b]{2}{*}{ Trauma patients } & Number & Validated Percent \\
\hline & 68 & 94.4 \\
\hline \multirow[b]{2}{*}{$\begin{array}{l}\text { Time from trauma to } \\
\text { presentation (months) }\end{array}$} & Mean & SE \\
\hline & 40.0 & 7.0 \\
\hline Clinical presentation & Number & Validated Percent \\
\hline Pain & 3 & 4.1 \\
\hline Giving away & 3 & 4.1 \\
\hline Both & 67 & 91.8 \\
\hline Associated injuries & Number & Validated Percent \\
\hline Lateral meniscus & 16 & 21.6 \\
\hline Medial meniscus & 28 & 37.8 \\
\hline Posterior cruciate ligament & 6 & 8.1 \\
\hline Types of graft & Number & Validated Percent \\
\hline Autologous & 32 & 43.2 \\
\hline Synthetic & 42 & 56.8 \\
\hline & Number & Validated Percent \\
\hline Postoperative complication & 10 & 13.5 \\
\hline
\end{tabular}


The abundant use of the synthetic graft in that period was observed in comparison to the practice nowadays. We used synthetic grafts in 42 individuals (56.8\%). The BTB was utilized four times and the Hamstring's was used in 28 individuals.

The Lachman test was positive in all patients preoperatively. Postoperatively, it decreased in magnitude but was still present in all knees reconstructed by synthetic ligaments. However, it was negative in 26 patients $(81.3 \%)$ and decreased in 6 patients in knees reconstructed using natural grafts (see Table 2).

The pivot shift test was done preoperatively and postoperatively. It was positive in all injured knees. Postreconstruction, it remains positive in 10 knees reconstructed by synthetic ligaments $(23.8 \%)$, while it was absent in all knees reconstructed by natural grafts (see Table 2). Obviously, the change in both tests between the two groups is significant ( $\mathrm{p}$ value $<0.05$ ).

The IKDC score scales from 0 to 100 . It consists of 18 items. It measures the functional outcome and the ability to return to the pre-injury level of activities. Each patient was assessed using this score pre- and postoperatively. The change of the score pre- and post-reconstruction was measured. Then, the average of the scores was obtained for knees reconstructed using the synthetic ligament or natural grafts. The prereconstruction IKDC average score for patients who underwent synthetic ligament reconstructions was 42. The post-reconstruction IKDC score for the same group was 67 . The average score changed from 42 to 67 (25 increase in the scale). The pre-reconstruction IKDC average score for patients who underwent natural ligament reconstructions was 44 . The post-reconstruction IKDC average score for the same group was 86 The average score changed from 44 to 86 (42 increase in the scale; see Table 3). Again, the change difference between the 2 groups is significant and the p value is less than 0.05 .

The KOOS score subjectively measure symptoms, pain, activity of daily living, quality of life and function in sports recreation. The sport subscale was the lowest one preoperatively. Thus, it is improved remarkably postoperatively. Pain was improved moderately as well as daily activities. All parameters saw greater improvement in natural ligament reconstructed knees than artificial ligament reconstructed knees, except sport recreation which was almost equal in both groups.

Ten individuals had complications after the ACL reconstruction surgeries. The main complication was the rupture of the graft. Four of them underwent Hamstring's tendons graft and 6 from the synthetic graft group $(\mathrm{P}>0.05$; see Table 4). However, 10 of the individuals who had synthetic ABC grafts complained of stiffness that was revealed to be due to an inflammatory reaction against the graft materials with recurrent synovitis which increased the risk of developing osteoarthritis (see Figure 1). Synthetic grafts were used for the 4 patients whose biological grafts ruptured. One patient underwent an autograft reconstruction which was complicated by rupture after an epileptic seizure, they then underwent a synthetic graft reconstruction which was again complicated by rupture after a seizure.

\section{Discussion}

The ACL is the most commonly injured ligament in sports people. Anatomic ACL reconstruction has been considered a satisfactory surgical procedure in sports medicine, and the autograft has been commonly used for ACL repairs in the past. ${ }^{11}$ Autografts are more commonly used than allografts or synthetic grafts. Autograft options that are commonly used are BTB and hamstring muscles. ${ }^{13}$ In recent decades the use of allograft is increasing. However, the

Table 2 Comparison Between Synthetic and Natural Grafts in Terms of Immediate Postoperative Tests

\begin{tabular}{|l|l|l|l|}
\hline Test & $\begin{array}{l}\text { Synthetic Ligament Reconstructed Knees } \\
\mathbf{N}=\mathbf{4 2}\end{array}$ & $\begin{array}{l}\text { Natural Grafts Reconstructed Knees } \\
\mathbf{N}=\mathbf{3 2}\end{array}$ & P value \\
\hline Lachman test remains positive & $42(100 \%)$ & $6 / 32(18.8 \%)$ & $<0.05$ \\
Pivot shift test remains & $10(23.8 \%)$ & $0(0 \%)$ & $<0.05$ \\
positive & & & \\
\hline
\end{tabular}

Table 3 Pre- and Post-Operative IKCD Scores for Both the Synthetic and Natural Grafts

\begin{tabular}{|l|l|l|l|l|}
\hline Type of Graft & Preoperative Score & Postoperative Score & Change in Score & P value for Change in Score \\
\hline Synthetic graft reconstructed knees & 42 & 67 & 25 & $<0.05$ \\
Natural graft reconstructed knees & 44 & 86 & 42 & \\
\hline
\end{tabular}


Table 4 Comparison of the Postoperative Complication Between Synthetic and Natural Graft

\begin{tabular}{|l|l|l|l|}
\hline & $\begin{array}{l}\text { Autologous, } \\
\text { N=32 }\end{array}$ & $\begin{array}{l}\text { Synthetic, } \\
\text { N=42 }\end{array}$ & P value \\
\hline $\begin{array}{l}\text { Presence of } \\
\text { Complication }\end{array}$ & $4(12.5 \%)$ & $13(31.0 \%)$ & $<0.05$ \\
\hline
\end{tabular}

synthetic grafts for reconstruction of ACL became popular in the 1980s, but after initial usage the utilization has been decreased due to poor mid-term results. ${ }^{17}$ In this study, we looked at the postoperative short- and long-term complications and found that the biological (autograft) was better in terms of clinical and functional outcomes than the synthetic autograft (the scores of KOOS and IKDC were better in natural grafts). Furthermore, the immediate postoperative results for both the pivot and Lachman tests were better in natural grafts. On the other hand, the rate of re-rupture was similar for both groups. However, the long-term inflammatory changes and stiffness that is attributed to the immunological reactions were higher in the synthetic grafts.

In a previous study about the BTB autograft, the longterm results (17-20 years) have shown $83 \%$ of patients having stable, normal or near normal functions while $1.6 \%$ of patients needed a revised ACL reconstruction. ${ }^{18}$ It has high strength and stiffness, consistency of the size of the graft, easy to harvest and can be secured well in the canal by interference screws. ${ }^{18}$ Complications include patellar
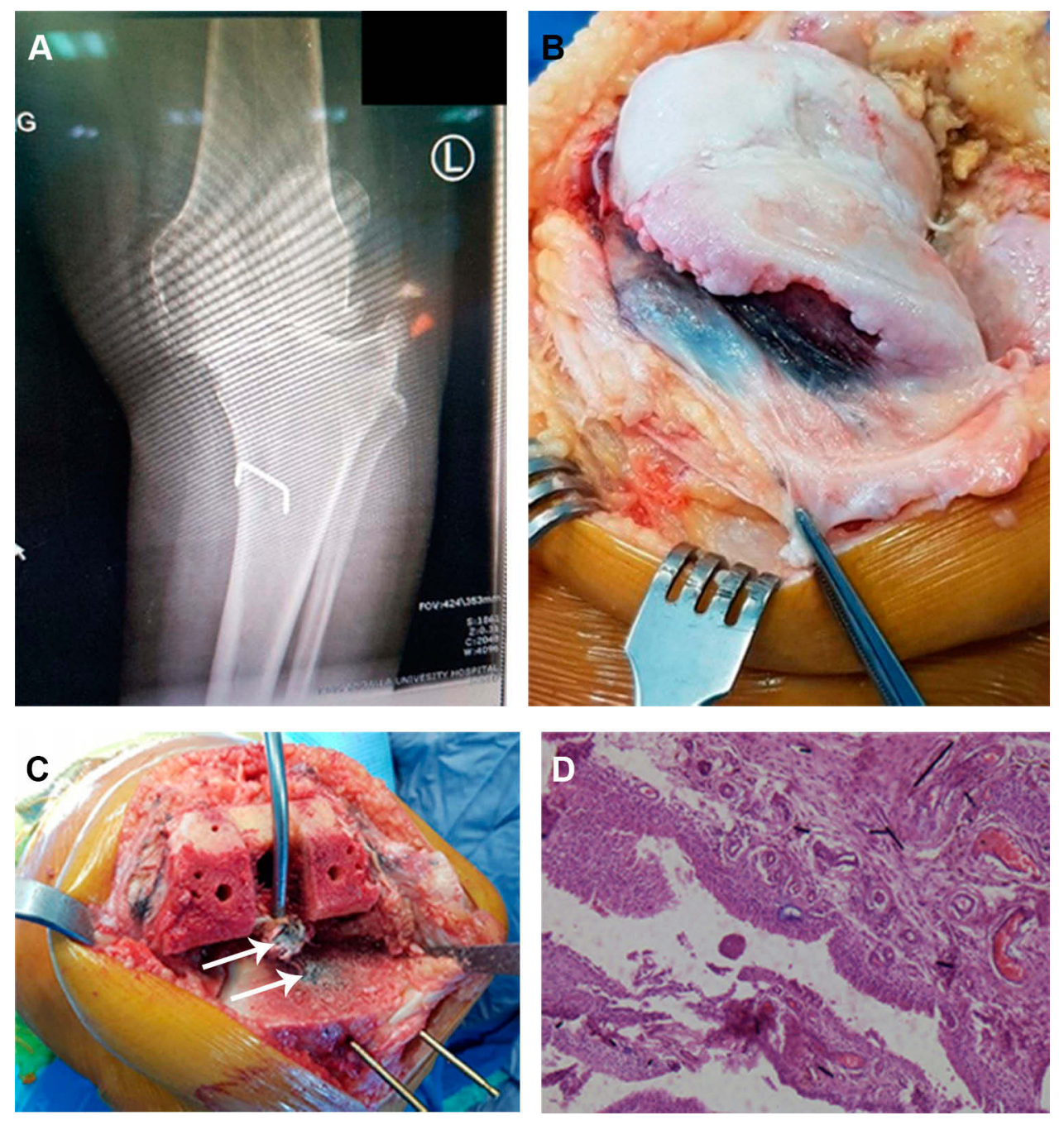

Figure I Total knee arthroplasty for a 54-year-old female patient with severe osteoarthritis of the knee joint. Notes: The patient was complaining of pain and recurrent synovitis 12 years post synthetic graft ACL-Reconstruction. (A) An X-ray showed the manifestation of severe osteoarthritis of the left knee. (B) Intraoperative black discoloration of the synovium around the femoral condyles area. (C) A remnant of the ABC synthetic graft (indicated by arrow) impeded in the tibia plateau and in the intercondylar area which was very difficult to remove. (D) Histopathology of the discolored synovium showed synovial hyperplasia with areas of fibrinoid necrosis and underlying mild chronic inflammation. Hemosiderin-laden macrophages are also seen. Several instances of black rod-shaped material is identified. There is no evidence of malignancy. 
tendon rupture, patellar/tibial fracture, quadriceps weakness, loss of full extension, anterior knee pain, and difficulty in kneeling and numbness due to injury of the infrapatellar branch of the saphenous nerve. ${ }^{18}$ Hamstring tendon grafts are one of the most commonly used grafts for ACL reconstruction. The semitendinosus tendon with or without gracilis tendon is harvested, from ipsilateral leg. ${ }^{18}$ The advantage of the hamstring graft is that there is no fear of fracturing the patella/tibial tuberosity, avulsion, and kneeling pain, thus minimizing donor site morbidity. The long-term follow-up results of hamstring grafts, up to 15 years, suggestthat $75 \%$ of patients scored normal or near normal results. ${ }^{19}$

Synthetic ligaments became popular in the 1980s and early 1990s. In one study, after analyzing 33 ruptured synthetic grafts with scanning electron microscopy, Mowbray et al correlated the high incidence of early prosthetic ligament failures due to the abrasion of the ligament at the tibial tunnel exit. The authors found that synthetic implants are particularly vulnerable to rupture if an impingement occurs. ${ }^{20}$ A study by Seitz demonstrated that the inflammatory reaction caused by wear particles of a foreign body leads to a peculiar modification in the composition of the synovial fluid. The depletion of the cartilage matrix that follows is responsible for the development of osteoarthritis. ${ }^{21}$

$\mathrm{Xu}$ et al had another opinion, they stated that the graft diameter is one of the factors that may influence the likelihood of success of the anatomic ACL repair, and it is also a key method of restoring the insertion site to at least $60 \%$ to $80 \%$ of the cross-sectional area. ${ }^{22}$ A pure autograft, however, provides superior results on the KT-1000 test and subjective evaluation. ${ }^{22}$ Genuario et al studied the cost-effectiveness for ACL reconstruction and they found that the hamstring muscle graft was the most cost-effective method for ACL reconstruction. ${ }^{23}$

In general, using synthetic grafts is associated with many problems. Tissue reaction from debris particulates can cause synovitis and knee pain. Although early failure of the ligament could be due technical errors, but obviously there is a high failure rate and poorer outcomes in synthetic grafts. ${ }^{8,9}$ On the other hand, using natural grafts is associated with less pain (no tissue reaction). Returning to preinjury levels of activities is excellent. Almost all-natural ligaments reconstructed knees return to their pre-injury level of activities. ${ }^{13}$ There were 4 patients with rupture of the ligament. Two of them were postepileptic seizures. Although the synthetic ligament avoids the harvesting site morbidity, it adds to the cost of the operation; artificial ligaments are costly. We did not encounter morbidities of the harvesting site other than simple hematomas and one infection. All were treated successfully.

This study has several limitations. First, it was a retrospective. Second, there was some difficulty in retrieving all patients with the low follow-up rate. There was a possibility of bias by involving patients with PCL injury and not only isolated ACL injury. Despite this, we think it was important, even with certain limitations, to revisit and present these data for the new generation of surgeons as evidence for synthetic graft experience to eliminate the potential return in the popularity of synthetic grafts for cruciate ligament reconstruction.

In conclusion, this study provided evidence that natural grafts are superior as they had better immediate and longterm postoperative clinical and functional outcomes. We recommend the utilization of autograft as a first choice, and the synthetic graft not to be used anymore, given the criteria. $\mathrm{TNw}$ generations of surgeon should be aware of synthetic grafts and their complications, and that many patients whoses grafts were synthetic may present in the future with complications. Also, patients with epilepsy should receive more care with regards to controlling their seizures, as they are more prone to orthopedic-related complications.

\section{Data Sharing Statement}

The data sets generated and analyzed during the current study are available from the corresponding author.

\section{Ethics and Patient Consent}

Institutional approval was obtained from the Institutional Review Board at Jordan University of Science and Technology. This study was conducted in accordance with the Declaration of Helsinki. No need for written consents due to the retrospective nature of the study. We confirm that the privacy of the participants was saved, and the data was anonymized and maintained with confidentiality. The patient data accessed complied with relevant data protection and privacy regulations.

\section{Funding}

This research did not receive any specific grant from funding agencies in the public, commercial, or not-forprofit sectors.

\section{Disclosure}

The authors report no conflicts of interest in this work. 


\section{References}

1. Daniel DM 1, Stone ML, Dobson BE, Fithian DC, Rossman DJ, Kaufman KR. Fate of the ACL-injured patient. A prospective outcome study. Am J Sports. 1994;22:632-644. doi:10.1177/ 036354659402200511

2. Fithian DC, Paxton LW, Goltz DH. Fate of the anterior cruciate ligament-injured knee. Orthop Clin North Am. 2002;33(4):621-636. doi:10.1016/S0030-5898(02)00015-9

3. Bonasia DE, Amendola A. Graft choice in ACL reconstruction. In: Bonnin M, Amendola A, Bellemans J, MacDonald S, Ménétrey J, editors. The Knee Joint. Paris: Springer; 2012:173-181.

4. Reinhardt KR, Hetsroni I, Marx RG. Graft selection for anterior cruciate ligament reconstruction: a level I systematic review comparing failure rates and functional outcomes. Orthop Clin North Am 2010;41(2):249-262. doi:10.1016/j.ocl.2009.12.009

5. Legnani C, Ventura A, Terzaghi C, Borgo E, Albisetti W. Anterior cruciate ligament reconstruction with synthetic grafts. A review of literature. Int Orthop. 2010;34(4):465-471. doi:10.1007/s00264-0100963-2

6. Ventura A, Terzaghi C, Legnani C, Borgo E, Albisetti W. Synthetic grafts for anterior cruciate ligament rupture: 19-year outcome study. Knee. 2010;17:108-113. doi:10.1016/j.knee.2009.07.013

7. Dhammi IK, Rehan-Ul-Haq KS. Graft choices for anterior cruciate ligament reconstruction. Indian $J$ Orthop. 2015;49:127-128 doi:10.4103/0019-5413.152393

8. West RV, Harner CD. Graft selection in anterior cruciate ligament reconstruction. J Am Acad Orthop Surg. 2005;13(3):197-207. doi:10.5435/00124635-200505000-00006

9. Zoltan DJ, Reinecke C, Indelicato PA. Synthetic and allograft anterior cruciate ligament reconstruction. Clin Sports Med. 1988;7:773-784

10. Makisalo S, Skutnabb K, Holmström T, Grönblad M, Paavolainen P. Reconstruction of anterior cruciate ligament with carbon fiber: an experimental study on pigs. Am J Sports Med. 1988;16(6):589-593. doi:10.1177/036354658801600606

11. Sherman $\mathrm{OH}$, Banffy MB. Anterior cruciate ligament reconstruction: which graft is best? Arthroscopy. 2004;20(9):974-980. doi:10.1016/ S0749-8063(04)00842-4

12. Prodromos CC, Joyce BT, Shi K. A meta-analysis of stability of autografts compared to allografts after anterior cruciate ligament reconstruction. Knee Surg Sports Traumatol Arthrosc. 2007;15 (7):851-856. doi:10.1007/s00167-007-0328-6
13. Crawford C, Kainer M, Jernigan D, et al. Investigation of postoperative allograft-associated infections in patients who underwent musculoskeletal allograft implantation. Clin Infect Dis. 2005;41(2):195-200. doi:10.1086/430911

14. Arnoczky SP, Warren RF, Ashlock MA. Replacement of the anterior cruciate ligament using a patellar tendon allograft: an experimental study. J Bone Joint Surg Am. 1986;68:376-385. doi:10.2106/000 04623-198668030-00010

15. Malinin TI, Levitt RL, Bashore C, Temple HT, Mnaymneh W. A study of retrieved allografts used to replace anterior cruciate ligaments. Arthroscopy. 2002;18(2):163-170. doi:10.1053/jars.2002. 30485

16. Petrou G, Chardouvelis C, Kouzoupis A, et al. Reconstruction of the anterior cruciate ligament using the polyester $\mathrm{ABC}$ ligament scaffold: a minimum follow-up of four years. J Bone Joint Surg Br. 2006;88:893-899. doi:10.1302/0301-620X.88B7.17671

17. Jackson DW, Grood ES, Goldstein JD, et al. A comparison of patellar tendon autograft and allograft used for anterior cruciate ligament reconstruction in the goat model. Am J Sports Med. 1993;21:176-185. doi:10.1177/036354659302100203

18. Chechik O, Amar E, Khashan M, Lador R, Eyal G, Gold A. An international survey on anterior cruciate ligament reconstruction practices. Int Orthop. 2013;37(2):201-206. doi:10.1007/s00264-0121611-9

19. Shaerf DA, Pastides PS, Sarraf KM, Willis-Owen CA. Anterior cruciate ligament reconstruction best practice: a review of graft choice. World J Orthop. 2014;5:23-29. doi:10.5312/wjo.v5.i1.23

20. Mowbray MAS, McLeod ARM, Barry M, Cooke WD, O’Brien TK. Early failure in an artificial anterior cruciate ligament scaffold. Knee. 1997:4:35-40. doi:10.1016/S0968-0160(96)00225-6

21. Seitz H, Marlovits S, Schwendenwein I, Müller E, Vécsei V. Biocompatibility of polyethylene terephthalate (Trevira-hochfest) augmentation device in repair of the anterior cruciate ligament. Biomat. 1998;19:189-196. doi:10.1016/S0142-9612(97)00201-9

22. Xu H, Lin W, Jin G, et al. Graft choice for anatomic anterior cruciate ligament reconstruction: the comparison between thin autograft and thick hybrid graft. An observational study. Medicine (Baltimore). 2018;97:e11597. doi:10.1097/MD.0000000000011597

23. Genuario JW, Faucett SC, Boublik M, Schlegel TF. A cost-effectiveness analysis comparing 3 anterior cruciate ligament graft types: bone-patellar tendon-bone autograft, hamstring autograft, and allograft. Am J Sports Med. 2012;40(2):307-314. doi:10.1177/ 0363546511426088
Orthopedic Research and Reviews

\section{Publish your work in this journal}

Orthopedic Research and Reviews is an international, peer-reviewed, open access journal that focusing on the patho-physiology of the musculoskeletal system, trauma, surgery and other corrective interventions to restore mobility and function. Advances in new technologies, materials, techniques and pharmacological agents are particularly welcome. The manuscript management system is completely online and includes a very quick and fair peer-review system, which is all easy to use. Visit http://www.dovepress.com/testimonials.php to read real quotes from published authors. 